\title{
Anatomical landmarks for the intercostal nerve blocks: A cross sectional cadaveric study
}

Mathangasinghe $\mathrm{Y}^{1}$, Samaranayake UMJE${ }^{1}$, Wijayasinghe $\mathrm{R}^{2}$, Manchanayake $\mathrm{M}^{2}$, Seneviratne $\mathrm{PD}^{2}$, Dassanayake $\mathrm{VEG}^{3}$

${ }^{\prime}$ Department of Anatomy, Faculty of Medicine, University of Colombo, ${ }^{2}$ Faculty of Medicine, University of Colombo.

${ }^{3}$ Department of Surgery, Faculty of Medicine, University of Colombo.

In ultrasound guided intercostal nerve blocks the nerve is often not observed despite the ability to visualize the neurovascular plane. The objective of this study was to identify the location of the intercostal nerve in relation to the lower border of the corresponding rib at the plane where intercostal nerve block is delivered. In this cross-sectional study, phenoxymethanol fixed cadaveric thoracic walls were frozen and parasagittal sections were made $7 \mathrm{~cm}$ from the mid-sagittal plane. Images were analysed using Image J (version-1.52a).Ninety-one intercostal (including subcostal) spaces of twelve cadaveric hemithoraces $[n=6$ (right), $\mathrm{n}=6$ (left)] were studied. The typical neurovascular pattern, i.e. vein(V), artery(A) and nerve $(\mathrm{N})$ lying from above downwards in VAN order was seen only on $37 / 91(40.7 \%)$ intercostal spaces. This was followed by the patterns NVA $(n=27 / 91,29.7 \%)$ and VNA ( $n=12 / 91,13.2 \%)$. A binary logistic regression was conducted to assess the determinants of the neurovascular pattern (dichotomous outcome variable="VAN-pattern" or "other-pattern"). The model with five predictors (sex, numerical value of the intercostal space, waist circumference, length of upper segment and shoulder width) was not statistically significant $[\chi 2(5, \mathrm{n}=91)=19.12, \mathrm{p}=.059]$. The nerve was located $4.4 \pm 1.7 \mathrm{~mm}$ deep and $0.5 \pm 2.6 \mathrm{~mm}$ inferior to the lower border of the corresponding rib. In conclusion, the typical intercostal neurovascular pattern (VAN) was observed only in $41 \%$ of the intercostal spaces, while the presence of this pattern was not predicted by the investigated independent variables. We recommend inserting the anaesthetic needle perpendicular to the skin, immediately below $(\sim 0.5 \mathrm{~mm})$ the corresponding rib. Clinical correlations are necessary for validation of these results. 